\title{
Transient and Spontaneous Idiopathic Factor V Inhibitor
}

\author{
Damian A. Laber*, Leela Bhupalam and Goetz H. Kloecker
}

\begin{abstract}
Division of Hematology and Medical Oncology, Department of Medicine, University of Louisville, James Graham Brown Cancer Center, Louisville, KY, USA
\end{abstract}

\section{TO THE EDITOR}

Since the first description in 1958, few cases of spontaneous factor V (FV) inhibition by an autoantibody have been described in the English medical literature [1,2]. Knowledge about the clinical implications of idiopathic FV inhibitor (FVI) is limited, but its recognition is important to prevent potential consequences.

We present the case of a 70-year-old woman found to have a prothrombin time (PT) of 26.8 seconds (s) and an activated partial thromboplastin time (PTT) of $71 \mathrm{~s}$ prior to an elective endoscopic retrograde cholangiopancreatography for symptomatic choledocholithiasis. She had no personal or family history of bleeding, thrombosis, autoimmune diseases, miscarriages, recent surgery or antibiotic use, and was not anticoagulated. Laboratory studies revealed a hemoglobin of $11 \mathrm{~g} / \mathrm{dl}$, and normal leucocyte count, platelet count, serum protein immunoelectrophoresis, D-dimer, fibrinogen, lupus anticoagulants and anticardiolipin antibodies. PT and PTT mixing studies revealed an inhibitor of a coagulation factor in the common pathway. Factor assays II, VIII and IX, were normal. Factor VII and X, both more than $50 \%$, were difficult to quantify secondary to an inhibitor effect in the system, with increasing activity after dilution. Factor $\mathrm{V}$ had $5 \%$ activity. Factor V inhibitor was 1.6 Bethesda Units. Prednisone $60 \mathrm{mg}$ was started and the ERCP successfully performed with no bleeding complication. Within 6 months the PT, PTT and factor V activity normalized and prednisone was tapered off.

Idiopathic FVI appear to be a rare occurrence compared with other secondary forms due to antibiotics, exposure to bovine FV, surgery, post transfusion, auto-immune diseases,

*Address correspondence to this author at the Division of Hematology and Medical Oncology, Department of Medicine, University of Louisville, James Graham Brown Cancer Center, Louisville, KY, USA; E-mail: damianla@ulh.org certain infections, pregnancy, malignancy and myeloproliferative disorders. Its diagnosis is made after excluding all known etiologies, making these cases difficult to confirm. Only one additional patient has been reported since a review of the cases between 1955 and 1997 [2,3].

Spontaneous FVI are auto-antibodies directed against FV. Inhibitors can bind either to a functional area of the factor, thus preventing it from participating in coagulation, or to a non-functional area causing degradation and/or removal of the molecule from circulation. FV circulates in plasma and also bound to platelets. Studies from a patient with FVI suggested that platelet-bound FV was relatively protected from an anti-FV antibody in whole blood, even though plasma FV was completely neutralized [4]. In another patient with FVI, residual FV was demonstrated in the platelet suspension [5].

We speculate that the lack of hemorrhage in our patient might be related to the poor accessibility of the antibody to platelet FV, leaving enough FV for effective hemostasis at the areas where the platelets go. The low rate of complications might be one of the reasons why idiopathic FVI is so rarely reported in the literature. Our case represents a well documented occurrence of a transient and spontaneous idiopathic FVI with no bleeding complication, and adds to the limited knowledge about this rare condition.

\section{REFERENCES}

[1] Ferguson J, Johnston Jr C, Howell D. A circulating inhibitor (AntiAcG) specific for the labile factor $\mathrm{V}$ of the blood clotting mechanism. Blood 1958; 13: 382-97.

[2] Knobl P, Lechner K. Acquired factor V inhibitors. Baillieres Clin Haematol 1998; 2: 305-18.

[3] de Raucourt E, Barbier C, Sinda P, Dib M, Peltier JY, Ternisien C. High-dose intravenous immunoglobulin treatment in two patients with acquired factor V inhibitors. Am J Hematol 2003; 74: 187-90.

[4] Nesheim ME, Nichols WL, Cole TK, et al. Isolation and study of an acquired inhibitor of human coagulation factor V. J Clin Invest 1986; 77: 405 .

[5] Bayani N, Rugina M, Haddad-Vergnes L, Lelong F. High-titer acquired factor $\mathrm{V}$ inhibitor responsive to corticosteroids and cyclophosphamide in a patient with two malignant tumors. Am J Hematol 2002; 71: 33-6. 